\title{
Implementando uma ferramenta para Monitorar as chuvas através da percepção das pessoas
}

\author{
Dacy C. Lobosco ${ }^{1}$, Paulo V. R. Carvalho ${ }^{1,2}$ \\ ${ }^{1}$ Sistemas de Informação - CEFET-RJ - Campus Nova Friburgo. \\ ${ }^{2}$ Programa de Pós-Graduação em Informática (PPGI) - UFRJ \\ dacy_lobosco@yahoo.com.br, paulov195617@gmail.com
}

\begin{abstract}
Municipal civil defense departments have great difficulties in predicting the impacts of rainfall due to the limitations of existing monitoring systems and the growing number of vulnerable communities. The article describes the construction of a mobile application based on the Design Science Research methodology that seeks to share information about the rains between the people of the city and the civil defense team. The results showed that the data from human sensors presented to civil defense agents are of great importance for emergency response actions.
\end{abstract}

Resumo. As secretarias de defesa civil municipais têm grandes dificuldades em prever os impactos das chuvas devido às limitações dos sistemas de monitoramento existentes e do crescente de comunidades vulneráveis. $O$ artigo descreve a construção de um aplicativo celular com base na metodologia Design Science Research que busca compartilhar informações sobre as chuvas entre as pessoas da cidade e a equipe de defesa civil. Os resultados mostraram que os dados apresentados aos agentes de defesa civil por pessoas em comunidades são de grande importância para as ações de resposta a emergências.

\section{Introdução}

A Zona de Convergência do Atlântico Sul, ZCAS na forma geralmente referida, é o principal sistema meteorológico de verão no Brasil responsável por um período prolongado de chuvas frequentes e volumosas em parte das regiões Norte, Centro-Oeste e Sudeste, pois carreia a umidade da região norte para a sudeste. Outro fenômeno climático recorrente são as frentes frias que atingem as regiões sul e sudeste do Brasil o ano todo, cerca de três a cinco vezes por mês. As frentes frias estão associadas ao aumento de nuvens, precipitação, variações de vento e fortes mudanças de temperatura. A combinação frequente deste 2 sistemas, principalmente no verão, traz chuvas fortes para região sudeste e, consequentemente para a região serrana do Rio de Janeiro.

A região serrana do Rio de Janeiro tem grande parte da população vivendo em torno de encostas e na cidade de Nova Friburgo a situação não é diferente. Em Nova Friburgo, em cerca de 50 anos, a população praticamente triplicou em um espaço territorial delimitado por cadeias de montanhas e rios (GASPARINI, 2012). Com isso 
houve desmatamento e ocupação desordenada das encostas que circundam a cidade. Essas ações desordenadas levaram a região a ser considerada como uma área de risco, com algumas áreas sujeitas a deslizamentos de terra e outras sujeitas a inundações (AGENDA 21 NOVA FRIBURGO, 2011).

Em 11 de janeiro de 2011, a região serrana do Rio de Janeiro foi atingida por uma tempestade que trouxe consequências drásticas para a região. A tempestade foi causada pela entrada de massas de ar provenientes da Zona de Convergência do Atlântico Sul (ZCAS) aliado a frentes frias procedentes do sul, fato considerado um evento normal de verão. Esse fenômeno pode ser observado na figura 1.

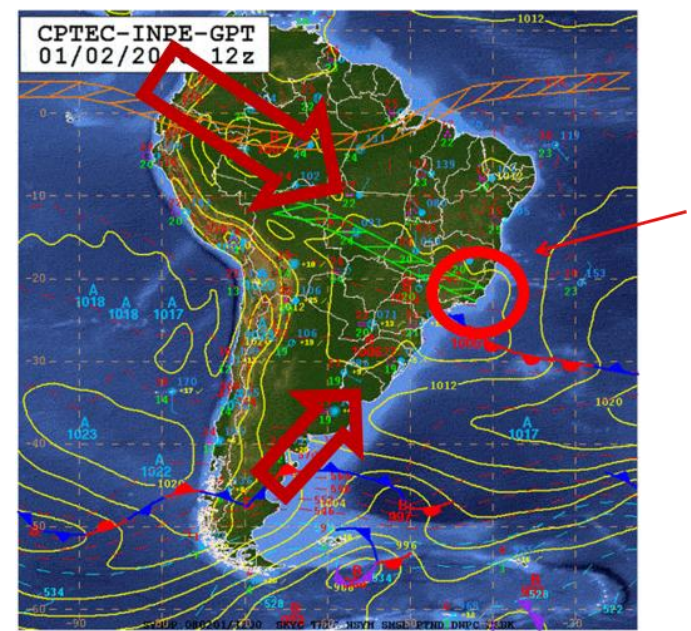

Figura 1. Zona de Convergência do atlântico Sul associado a uma frente Fria

Em 2011, a grande quantidade de chuvas associada ao crescimento populacional e ao uso e ocupação incorretos do solo, com edifícios em locais de risco e mal estruturados, associado a erosões de leitos de rios, culminaram em grandes deslizamentos e inundações em massa na região serrana do Rio de Janeiro, com 864 mortes e 22.479 desabrigados. Entre todas as cidades atingidas pelo desastre de 2011, Nova Friburgo foi a que apresentou o maior número de mortes e uma maior extensão territorial alcançada pelo desastre. (BANCO MUNDIAL, 2012).

Nos relatórios e relatos da comunidade, os movimentos de massa e a tempestade ocorreram de maneira diferente em cada localidade de Nova Friburgo. Diante desse cenário, pode-se observar que a percepção da população em relação à chuva também foi modificada e contextualizada de acordo com a experiência vivida em cada localidade da cidade. Isso significa que a mesma intensidade de chuva de $50 \mathrm{~mm}$, é percebida como uma chuva de forte intensidade numa localidade e para outra localidade, na mesma cidade, significa uma chuva de fraca intensidade ou moderada intensidade.

\section{Organização da Defesa Civil}

O Serviço de Defesa Civil foi criado em 1942 como Serviço de Defesa Antiaérea Passiva. (SECRETARIA NACIONAL DE DEFESA CIVIL, 2017). No início, as atividades de defesa civil visavam apenas minimizar as consequências de desastres, como as atividades de socorro pós-desastre (NETO, 2007). Atualmente, além 
dessas atividades, estão incluídas ações de prevenção para reduzir as consequências decorrentes desses eventos.

De acordo com o CEPED (Centro Universitário de Estudos e Pesquisas sobre Desastres ) / UFSC, 2012, as funções de defesa civil foram atualizadas pela Estratégia Internacional para Redução de Desastres e também mudaram no Brasil desde a publicação da Política Nacional de Proteção e Defesa Civil (PNPDEC). A Defesa Civil pode ser conceituada como um conjunto de ações preventivas, de mitigação, preparação, resposta e recuperação, destinadas a reduzir os riscos de desastres, com o objetivo de preservar o bem estar da população, restaurar a normalidade social e a proteção civil. Percebemos nessa conceituação que a ação de defesa civil tem como objetivo principal a redução de riscos e desastres, que inclui cinco ações distintas e inter-relacionadas, que são ações de: prevenção; mitigação; preparação; resposta; e recuperação. Essas ações ocorrem de maneira multissetorial e nos três níveis de governo (federal, estadual e municipal), exigindo ampla participação da comunidade para o seu efetivo sucesso.

\subsection{Monitoramento da Chuva no Brasil}

No Brasil, em julho de 2011, um decreto presidencial criou o Centro Nacional de Monitoramento e Alerta de Desastres Naturais (CEMADEN), uma agência vinculada ao Ministério da Ciência, Tecnologia, Inovação e Comunicações (MCTIC). O principal objetivo do CEMADEN é monitorar o clima e emitir alertas para reduzir a vulnerabilidade social, ambiental e econômica resultante desses eventos (CEMADEN, 2018). O CEMADEN foi criado em parceria com várias instituições para implementar, complementar e consolidar a rede de instrumentos meteorológicos, hidrológicos e geotécnicos de monitoramento ambiental.

Durante observações de campo na defesa civil de Nova Friburgo, analisamos os principais instrumentos meteorológicos utilizados pelos agentes para a previsão de chuva. Esses instrumentos são sites disponibilizados pelo CEMADEN ou por institutos governamentais parceiros em previsões meteorológicas. Os instrumentos mostram imagens de satélite com dados do passado recente e uma previsão do futuro, conforme mostrado nas figuras 2 e 3 . Com base nesses dados e nos avisos emitidos pelo CEMADEN, os agentes de defesa civil tomam decisões de emergência que impactarão na vida da população da cidade.

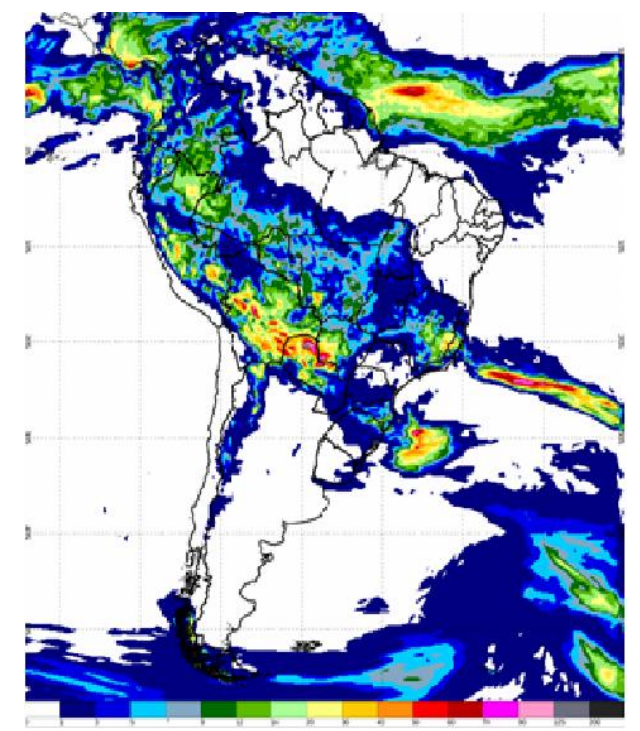

Figura 2. Dados do Passado imagem do satélite

Instituto Nacional de Meterologia INMET

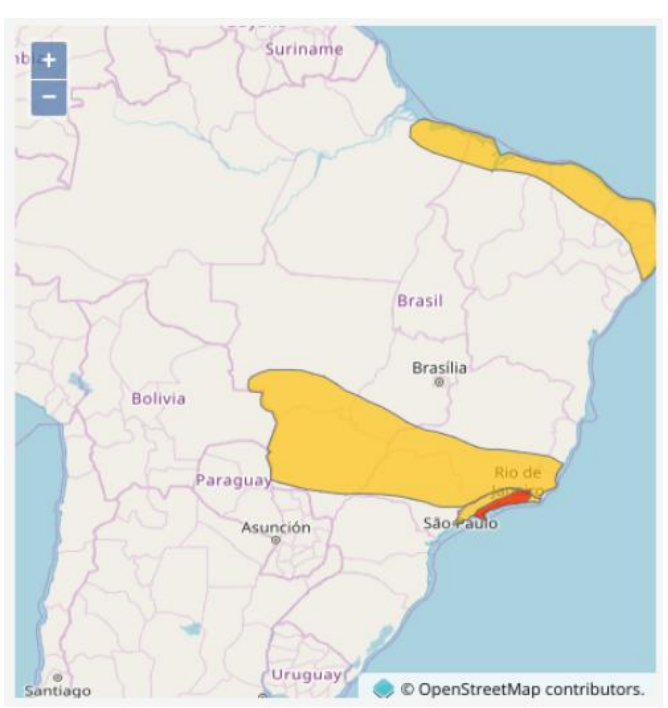

Figura 3. Alerta de tempestade Dados do Futuro

Centro de Previsão de Tempo e Estudos Climáticos - CPTEC/INPE 
Um aspecto interessante a ser observado é a qualidade das informações fornecidas pelo CEMADEN nas previsões e alertas. Em relação à qualidade das previsões e avisos, o CEMADEN (2017) nos diz que enfrenta limitações em relação às incertezas das previsões meteorológicas; problemas no diagnóstico da natureza dos fenômenos meteorológicos que operam em uma determinada área, principalmente em termos de duração e tamanho. Dificuldades para obter informações ininterruptas de precipitação pluviométrica, áreas com baixa densidade de pluviômetros que não fornecem dados do passado para cobrir uma área geográfica significativa, falhas e atrasos no recebimento de dados de chuva pela rede hidrológica, rede hidrológica ruim, imprecisão na descoberta dos parâmetros de precipitação em cada localidade de cada cidade para prever deslizamentos em massa, falha na obtenção de informações sobre movimentação de terra, condições das ruas, lixões, ralos entupidos, vazamentos de líquidos, condições de construção residencial, falhas na descoberta de chuvas que ocorrem em municípios vizinhos que afetam o município observado. Todos esses fatores indicaram que os agentes de defesa civil têm vários problemas em descobrir o que realmente ocorre no campo em tempo real e entender as situações de emergência.

Dolif, G. ET al. (2013) já observaram essas questões investigando os desafios dos agentes do ALERTA RIO na análise dos dados meteorológicos fornecidos pelo CEMADEN e outros institutos meteorológicos. O estudo de campo mostrou questões sobre integração de dados, desempenho de modelos meteorológicos e também a necessidade dos agentes em ter uma "janela para o exterior" que facilite seu trabalho de visualizar a chuva que está ocorrendo. Eles explicam que, olhando para o exterior, poderiam em tempo real validar dos dados computacionais (gráficos e previsões).

Tais problemas nos dados meteorológicos e suas análises, confirmados pelo CEMADEN e ratificados por observações de campo, indicaram que a tomada de decisões das equipes de defesa civil com base apenas nesses dados poderia ser melhorada com informações mais detalhadas sobre o que está acontecendo em campo, informações provenientes das comunidades locais em situações de chuva forte.

Além disso, analisando os relatórios da comunidade de Nova Friburgo após eventos de chuvas fortes, pode-se observar que há avisos frequentes de chuvas fortes que não ocorrem (falso-positivos) ou situações em que a comunidade não é avisada e chuvas fortes ocorrem chuvas (falso-negativos). Esses alertas inadequados ou falsos (positivos ou negativos) podem ser entendidos devido a problemas nos dados e infraestrutura meteorológicos e às incertezas naturais nessas medições. Uma nova estrutura de comando e controle (C2) que inclua formalmente os conselhos, avisos e percepções da população local, juntamente com formas de capturar, organizar e estruturar essas informações facilitaria o trabalho e a tomada de decisão das equipes de resposta a emergências.

Esta pesquisa procura preencher essa lacuna encontrando soluções tecnológicas sem grandes investimentos financeiros em infraestrutura e de maneira colaborativa e criativa para incluir todos os atores envolvidos que participam do processo da prevenção a resposta à emergências. A solução proposta visa, por um lado, disponibilizar informações de defesa civil de forma mais personalizada para cada localidade da cidade e, por outro lado, permitir que as equipes de defesa civil obtenham dados da população em tempo real, favorecendo os agentes e a tomada de decisão. 


\section{Metodologia}

Esta pesquisa segue o processo iterativo da Design Science Research (DSR), desde a descoberta de fatos até a conscientização sobre os problemas, planejamento de soluções / ações, design de artefatos, avaliação e reflexão sobre os problemas. A Design Science Research tem sido amplamente utilizada nas áreas de engenharia e sistemas de informação, visando o desenvolvimento e soluções de projeto para resolver classes de problemas, propor novos artefatos e propor melhorias ou alternativas às soluções existentes (Aken 2004; Hevner ET al., 2004). Segundo Aken (2004), a principal missão da Design Science é desenvolver conhecimento para o design e desenvolvimento de artefatos. Ele procura preencher a lacuna entre teoria e prática e estimular o rigor necessário para garantir a confiabilidade nos resultados alcançados (Hevner ET al., 2004).

O método é dividido em etapas altamente interconectadas e algumas vezes sobrepostas, de uma maneira que os resultados de alguma etapa influenciam na revisão de uma anterior. A fase de descoberta de fatos inclui revisão da literatura e estudos etnográficos de campo em uma pesquisa fundamentada que visa o desenvolvimento de artefatos aplicados e úteis. Os estudos de campo possibilitam o envolvimento de agentes da defesa civil e as pesquisas em uma construção social (Carvalho ET al., 2016) organizada conforme indicado na figura 4.

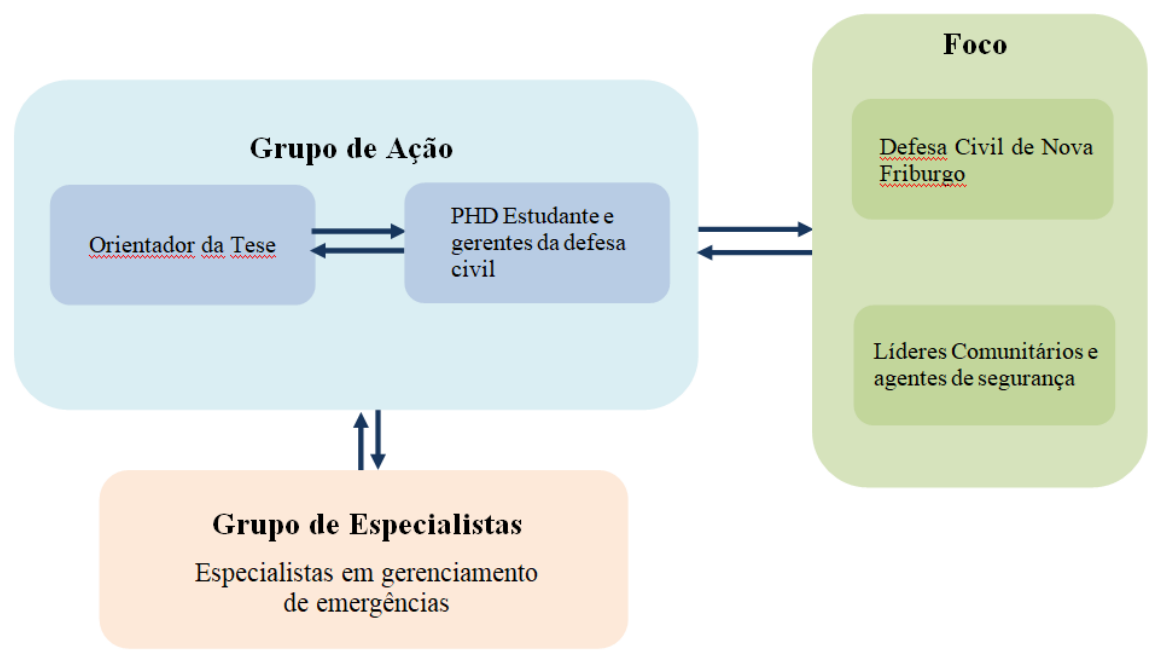

Figura 4. Construção Social

\section{Proposta de Solução}

O objetivo é tornar toda a população de Nova Friburgo uma rede de sensores humanos para compartilhar percepções sobre a intensidade e impacto das chuvas no seu local. Conforme (Goodchild, 2007), o objetivo é utilizar a inteligência e a experiência de cada pessoa na área em que vive, aproveitando o fato de que os seres humanos são sensores inteligentes capazes de fornecer dados relevantes para uma emergência. Os dados da população são compartilhados com as equipes de emergência por meio de um sistema colaborativo ${ }_{2}$ que disponibiliza entre os envolvidos informações sobre o real impacto das chuvas em cada localidade. As percepções da população irão preencher um mapa da situação das chuvas, mapa que será disponibilizado para a população e a 
equipe de emergência ao mesmo tempo, de maneira que ambos sejam capazes de analisar e avaliar o impacto das chuvas. A defesa civil poderá enviar alertas com dados das chuvas acumuladas e alertas com dados das previsões de acordo com as agências governamentais, e a população fornece a percepção da chuva em cada localidade. Essa interação pode ser observada na figura 5 , no qual visões mutuamente percebidas da situação estão sendo construídas em conjunto entre a população e os agentes de emergência.

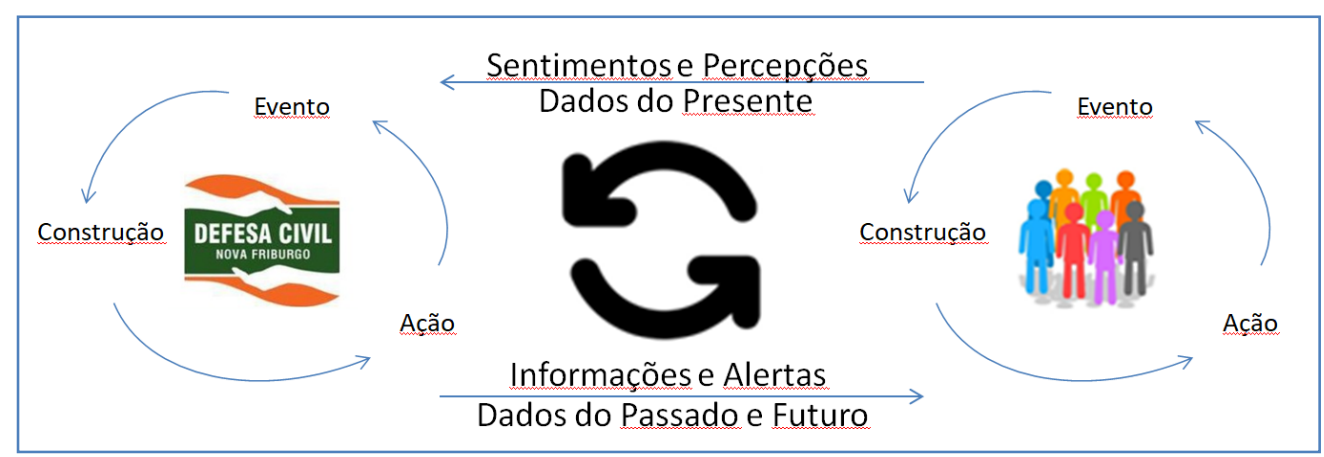

Figura 5: Construção de uma Visão comum sobre a Chuva

Um aplicativo foi desenvolvido de acordo com os conceitos de um sistema colaborativo para interação entre a população e os membros da equipe de defesa civil. Após o desenvolvimento, o sistema foi testado em situações reais de chuva. Os resultados obtidos foram comparados com as previsões dos órgãos oficiais, a fim de verificar a influência dos dados sobre a tomada de decisão dos agentes de Defesa Civil.

A principal forma de interação da população com a defesa civil é por meio de uma interface onde a população informa sua percepção de chuva. $O$ post que vem da população inclui a posição do GPS, o número do celular e a percepção da chuva. Na figura 5 abaixo, mostramos a interface do usuário (população) onde cada pessoa informa sua percepção da chuva. A intensidade da chuva está associada a uma cor, o que será importante para traçar informações para defesa civil e facilitar a análise cognitiva.

Do lado da Defesa Civil, sempre que houver uma atualização de informações importantes, os agentes devem informar a população. Tipos de atualizações: previsão de chuvas (dados futuros), níveis de alerta, situação das chuvas nas últimas horas (dados do passado), resposta a mensagens e dúvidas do público, informações, solicitação de evacuação para pontos de apoio, boletins de situação de emergência . A Figura 6 abaixo mostra a interface da população quando recebe uma informação ou alerta da defesa civil. 


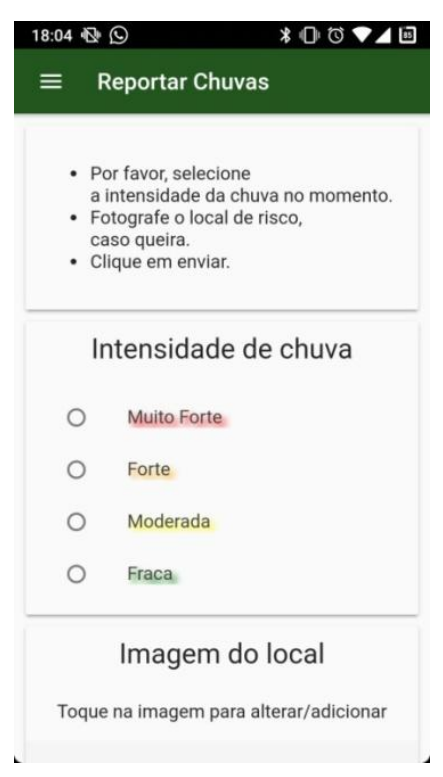

Figura 5. Chuva Report

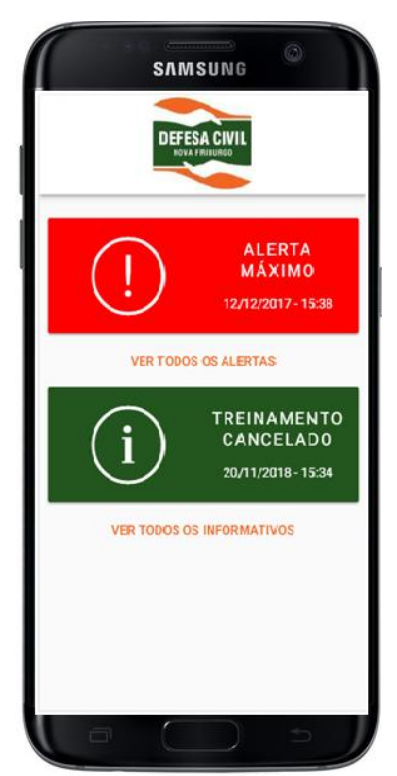

Figura 6. Alertas da Defesa Civil

A Figura 7 abaixo mostra como as percepções da população são visualizadas dentro do mapa. No aplicativo da cidade de Nova Friburgo, cada círculo representa uma pessoa como sensor no contexto da localidade da cidade informando a situação da chuva no momento em que está ocorrendo. Existem quatro tipos de intensidade disponíveis para a população informar sua percepção com suas respectivas cores: (Muito forte - vermelho, Forte - laranja, Moderado - amarelo, Fraco - verde). No mapa a cor da contribuição é visualizada.

Outra diferenciação é feita por meio dos símbolos, onde a população em geral recebe um círculo como símbolo. Voluntários treinados em defesa civil recebem o quadrado como símbolo e agentes de defesa civil recebem o triângulo como símbolo. Assim, ao olhar para o mapa, é possível diferenciar imediatamente a percepção da população, voluntários e agentes treinados.

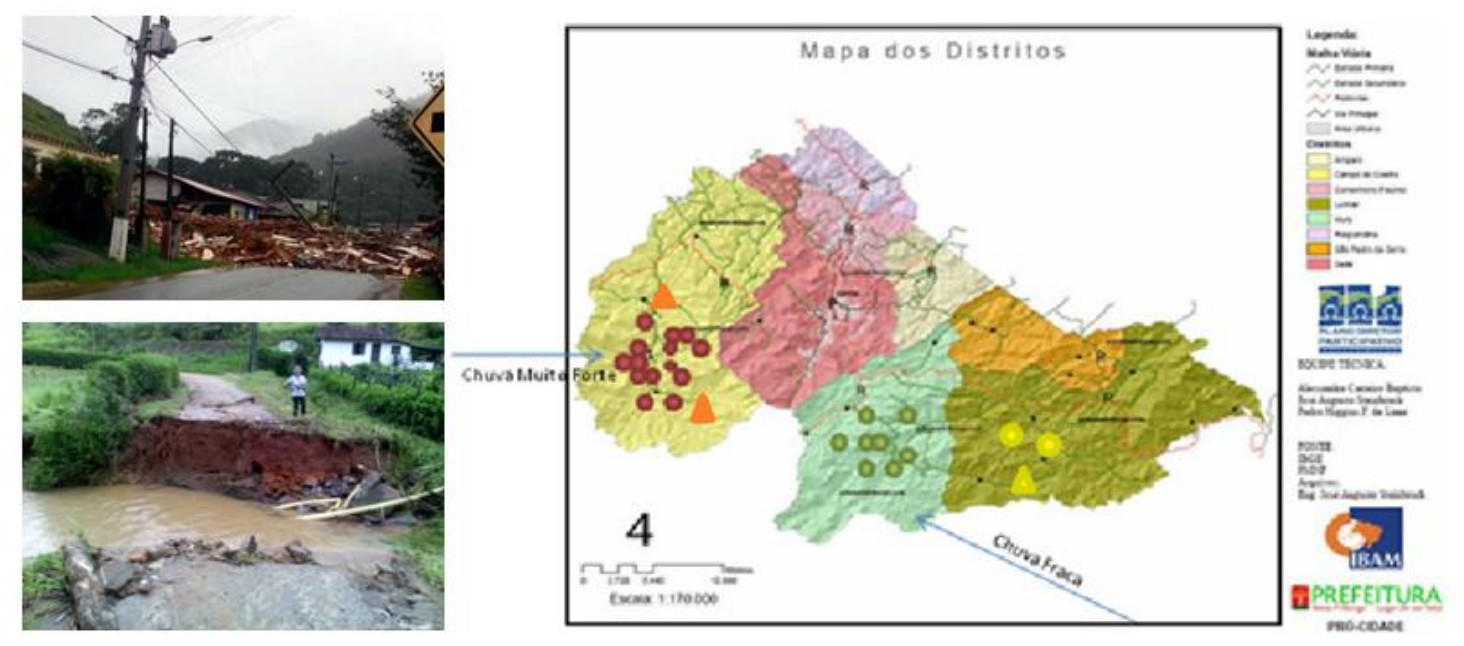

Figura 7. Mapa da Chuva 


\section{Testando a Solução}

Após o primeiro ciclo do DSR, a solução proposta foi avaliada em situações reais de chuva na cidade de Nova Friburgo. Agentes da defesa civil e voluntários das comunidades participaram dos testes. Este teste foi realizado por 14 dias entre janeiro e fevereiro de 2019, período escolhido por ser um dos mais chuvosos do ano. Durante o período de testes foram armazenadas informações das previsões oficiais das agências e a percepção da chuva de voluntários e agentes por meio da ferramenta. A Figura 13 abaixo ilustra como é o mapa de ferramentas e como ele apresenta dados para as pessoas sobre sua contribuição na chuva. A Figura 13 é uma captura de tela durante o evento de chuva em 31/01/2019.

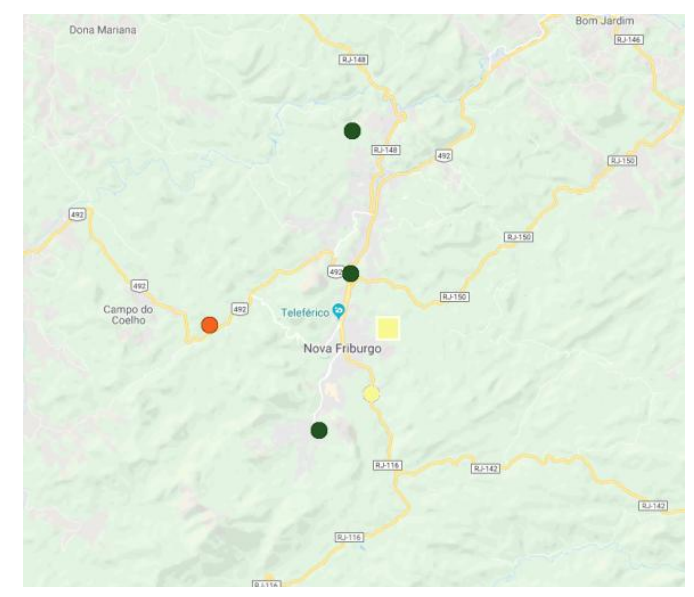

Figura 8. Mapa do aplicativo com as percepções em situação real. (31/01/2019)

Uma contribuição importante que podemos destacar a partir da captura de tela da figura 8, em comparação com a previsão, é a de que a previsão sempre fornece um cenário único do pior caso para toda a cidade, e a ferramenta durante os testes fornece dados com a intensidade da chuva de cada localidade da cidade. Para fins de comparação entre os dados de previsão e os dados do aplicativo, usamos o pior caso ocorrido em cada dia durante o período de teste. Durante os 14 dias de testes, a figura 9 compara as previsões das agências e a contribuição dos voluntários por meio do aplicativo:

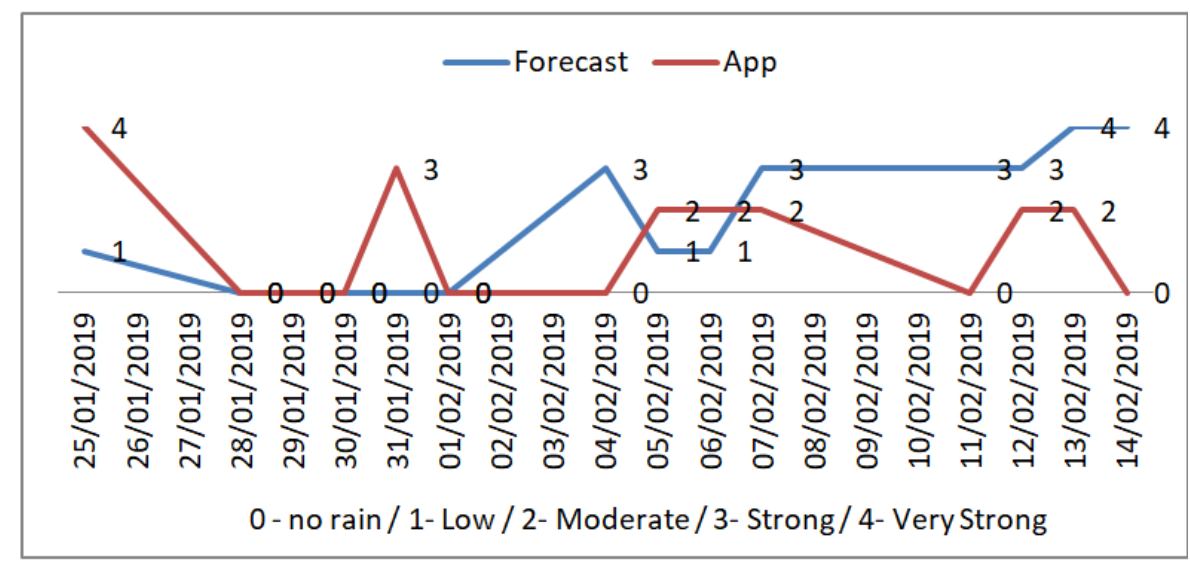

Figura 9. Comparativo das previsões com os dados da ferramenta 


\section{Conclusão}

As Figuras 8 e 9 mostram um aumento de informações e detalhes para a tomada de decisão de agentes da defesa civil que não existiam anteriormente com instrumentos oficiais. Isso pode ser identificado pelas cores diferentes da intensidade das chuvas dos sensores humanos distribuídos pela cidade. E essas são mais variáveis que alimentam de informação a equipe de emergência e que podem auxiliar tomadas de decisão e ações de resposta.

A pesquisa apresenta uma estrutura para orientar o desenvolvimento de uma solução sociotécnica que possibilite a conscientização da situação da chuva além de simples troca de mensagens, e informações, bem como a visualização de contribuições em um mapa. O mapa e as mensagens são vias criadas pela ferramenta para compartilhar as percepções da chuva entre a população e os agentes. Com a definição da solução, ela foi posta em prática durante um teste com voluntários da defesa civil, e os resultados obtidos por meio de entrevistas e questionários mostram que as informações colaborativas e compartilhadas são de grande valia para melhorar a consciência da situação dos agentes. O relato de todas as pessoas envolvidas indicou que o uso de seres humanos como sensores de chuva pode ser a janela que faltava para que os agentes tenham uma visão geral da situação local das chuvas e um panorama da percepção da população sobre o impacto das chuvas.

\section{Referências}

AGENDA 21 NOVA FRIBURGO (2011). River Action Plan: Agenda 21 document of Nova Friburgo. Action plans for sustainable development. ISER. Nova Friburgo, RJ.

Aken, J. E. V. (2004). "Management Research Based on the Paradigm of the Design Sciences: The Quest for Field-Tested and Grounded Technological Rules." Journal of Management Studies 41 (2): 219-246.

BANCO MUNDIAL (2012). Loss and Damage Assessment: Floods and Landslides in the Rio de Janeiro Mountain Region - January 2011. Report prepared by the World Bank with the support of the Rio de Janeiro State Government.

CARVALHO, P. V. R.; Huber G. ; RIGHI, A. W. ; LEMOS, C. ; EMYGDIO, K. ; Gomes, José Orlando (2016). Firefighting emergency response exercise ? an analysis of standardization and resilience. In: 13th International Conference on Information Systems for Crisis Response and Management, 2016, Rio de Janeiro. ISCRAM 2016 Conference Proceedings. Bruxelas: ISCRAM, 2016.

CEMADEN (2017). Proceedings of the 1st National Cemaden Alert Assessment Seminar. Sao Jose dos Campos.

CEMADEN (2018). National Center for Natural Disaster Monitoring and Alerts. Available at: $<$ http://www.cemaden.gov.br $>$. Accessed on: Jan 10th.

CEPED / UFSC (2012) - University Center for Disaster Studies and Research / Federal University of Santa Catarina. Basic training in Civil Defense. Florianópolis: CAD UFSC, 2012. 
Dolif, E., Engelbrecht, A., Jatobá, A., Silva, A., Gomes, J., Borges, M., Nobre, C., Carvalho, P. (2013); Resilience and brittleness in the ALERTA RIO system: a field study about the decision-making of forecasters. Brasil: Rio deJaneiro, RJ, 2013.

GASPARINI, MARINA FAVRIM (2012), Rural work, health and social and environmental contexts - a case study on the perception of risks associated with flower production in agricultural communities in Nova Friburgo, RJ. (DISSERTATION), Sergio Arouca National School of Public Health (ENSP / FIOCRUZ). Rio de Janeiro; s.n; 2012. 69 p.

Goodchild, M. (2007). Citizens as sensors: the world of volunteered geography. GeoJournal 69 (4): 211-221.

Hevner, A. R., S. T. March, J. Park, and S. Ram (2004). "Design Science in Information Systems Research.” MIS Quarterly 28 (1): 75-105.

NETO, M. C (2007). Legal aspects of Civil Defense activities. Ministry of National Integration. National Secretariat of Civil Defense.

SECRETARIA Nacional de Defesa Civil (2017). Disponivel em http://www.defesacivil.gov.br/glossario/index1.asp. Accessado em: 10-2017. 